\title{
CHEMOTHERAPY IN A PATIENT WITH PRIOR HISTORY OF IDIOPATHIC THROMBOCYTOPENIC PURPURA
}

\author{
Kouji Kanemoto, Hiroaki Satoh, Kiyohisa Sekizawa \\ University of Tsukuba: Division of Respiratory Medicine, Institute of Clinical Medicine
}

\begin{abstract}
Summary: We described a 67 years old small-cell lung cancer patient with a prior history of idiopathic thrombocytopenic purpura (ITP) who was treated with successful chemotherapy. It is probably safe to administer chemotherapeutic agents for some cancer patients with prior history of ITP, but it is important to prevent or minimize the toxicities of these chemotherapeutic agents.
\end{abstract}

Key words: Idiopathic thrombocytopenic purpura

\section{Introduction}

The association of idiopathic thrombocytopenic purpura (ITP) with malignant diseases of the lymphoid system is common $(1,2,4,5)$, however, non lymphoid malignancies associated with ITP have also been observed; lung cancer has been one of the most common solid tumors reported $(3,7,9,10)$. We show a lung cancer patient with prior history of ITP who was treated with successful chemotherapy.

\section{Case report}

A 67-year-old man was admitted because of productive cough. The patient had a prior history of ITP 6 years previously. The chest x-ray revealed a mass in the left lung with mediastinal lymph node adenopathy. Performance status (ECOG) (11), of the patient was 1 . on admission, the platelet associated $\mathrm{IgG}$ was increased $(62.7 \mathrm{ng} / 107$ cells) (normal range: $0-25 \mathrm{ng} / 107$ cells), while thrombocyte count was 274 x 109/L. Transbronchial biopsy of the tumor was performed, and histologic evaluation revealed a diagnosis of small cell lung cancer (SCLC). Examination by systemic survey revealed no metastases. The patient was informed about the diagnosis and the risk associated with chemotherapy. However, the patient agreed to receive chemotherapy, then he underwent two courses of chemotherapy with carboplatin (area under the curve: $5 \mathrm{mg} / \mathrm{ml}$ per minute, Calvert formula (8), day 1$)$ and etoposide $\left(100 \mathrm{mg} / \mathrm{m}^{2}\right.$, days 1 , 2 , and 3 ) underwent. The response to the therapy was evaluated as a good partial response. Thereafter the patient received 2 other courses of the chemotherapy with thoracic irradiation (70 Gy) concurrently. In each course of the chemotherapy, the patient did not develop grade 3-4 myelosuppression (NCI toxicity criteria). Two months after the end of the chemoradiotherapy, the patient developed lumbar bone metastasis, which was treated with irradiation successfully. He is still alive 12 months from the beginning of the chemotherapy without any episode of ITP, although the elevated level of platelet associated $\mathrm{IgG}$ continues (62.8 ng/107 cells).

\section{Discussion}

Lung cancer, either SCLC or non-small cell lung cancer, may be associated a wide spectrum of paraneoplastic syndromes such as secondary endocrinopathies, neurpathies, and autoimmune thrombocytopenic purpura $(7,9,10)$. Because the elevated level of the platelet associated $\mathrm{IgG}$ was observed in our case, we could not exclude the possibility of ITP due to SCLC as paraneoplastic phenomenon. However, the patient had no medication for SCLC and ITP for 6 years, and platelet count at admission was within normal range. Therefore, we considered that the ITP in our patient might not have been due to SCLC.

Thrombocytopenia is a frequent comorbid condition in many patients. In some patients, drugs are the cause of low platelet counts. While cytotoxic effects of anti-tumor therapy are the most frequent cause, immune mechanism would also be considered. Demirer et al. reported a patient with SCLC developed autoimmune thrombocytopenic purpura, following a cyclophosphmide, paclitaxel-containig regimen for peripheral blood stem cell mobilization (3). More recently, Mitsuhashi et al showed the autoimmune thrombocytopenia in patients with carcinoma of the uteric cervix developing after cisplatin and radiation therapy (6). In these cases, post-chemotherapy immunologic dysregulation may be a trigger for the development of autoimmune thrombocytopenic purpura. Although there has been no direct 
data on showing that it is on immune basis, cytotoxic drugs can cause and recur ITP. We described the case of a SCLC patient with a prior history of ITP who needed the administration of anticancer drugs. To our knowledge, there have been no reports of chemotherapy for SCLC patients with a prior history of ITP in English literature. Our experience shows that it is probably safe to administer chemotherapeutic agents for some cancer patients with prior history of ITP, but it is important to prevent or minimize the toxicities of these chemotherapeutic agents. Fully consideration about the indication of chemotherapy and careful observation are required for such patients.

\section{References}

1. Ben-Yehuda D, Gillis S, Eldor A. Clinical and therapeutic experience in 712 Israeli patients with idiopathic thrombocytopenic purpura. Israeli ITP Study Group. Acta Haematol 1994;91:1-6.

2. Cohen JR. Idiopathic thrombocytopenic purpura in Hodgkin's disease: a rare occurrence of no prognostic signifgicance. Cancer 1978;41:743-6.

3. Demirer T, Celebi H, Arat M et al. Autoimmune thrombocytopenia in a patient with small cell lung cancer developing after chemotherapy and resolving following autologous peripheral blood stem cell transplantation. Bone Marrow Transplant 1999;24:335-7.

4. DiFino SM, Lachant NA, Kirschner JJ, Gottlieb AJ. Adult idiopathic thrombocytopenic purpura. Clinicla findings and response to therapy. Am J Med 1980;69:430-42.
5. Kim HD, Boggs DR. A symdrome resembling idiopathic trombocytopenic purpura in 10 patients with diverse forms of cancer. Am J Med 1979;67:371-7.

6. Mitsuhashi A, Yamazawa $\mathrm{K}$, Tanaka $\mathrm{N}$ et al. Autoimmune thrombocytopenia in women with carcinoma of the uterine cervix developing after cisplatin and radiation therapy. BJOG 2002;109:103-4.

7. Ohura $\mathrm{H}$, Sagawa M, Yoshida $\mathrm{H}$ et al. A case of idiopathic thrombocytopenic purpura combined with lung cancer. Kyobu Geka 1996;49:484-6.

8. Rosell R, Gatzemeier U, Betticher DC et al. Phase III randomized trial comparing paclitaxel/carboplatin with paclitaxel/cisplatin in patients with advanced non-smallcell lung cacer - a cooperative multinational trial. Ann Oncol 2002; 13:1539-49.

9. Tanita T, Nishimura T, Kubo H et al. A case of lung cancer combined with ocular myopathy and idiopathic thrombocytopenic purpura which was presumed to be cuased by heart catheterization. Kyobu Geka 1994;47:328-31.

10. Tsuchishima S, Shimizu T, Yuasa K et al. Lung cancer surgery in a patient with idiopathic thrombocytopenic purpura. Kyobu Geka 1998;51:424-7.

11. Zubrod G, Scheniderman M, Frei E et al. Appraisal of methods for the study of chemotherapy of cancer in man: comparative therapeuric trail of nitrogen mustard and triethlene thiophosphoramide. J Chron Dis 1960;11:7-33.

Submitted November 2002.

Accepted February 2003.

Hiroaki Satoh, M.D.,

Division of Respiratory Medicine, Institute of Clinical Medicine, University of Tsukuba, Tsukuba-city, Ibaraki, 305-8575 Japan.

e-mail: hirosato@md.tsukuba.ac.jp 